\title{
Excited-State Dynamics in the RNA Nucleotide Uridine 5'-Monophosphate Investigated using Femtosecond Broadband Transient Absorption Spectroscopy
}

\author{
Matthew M. Brister and Carlos E. Crespo-Hernández* \\ Department of Chemistry, Case Western Reserve University, 10900 Euclid Avenue, Cleveland, \\ Ohio 44106, United States; *Email: carlos.crespo@ case.edu
}

\section{Supplementary Information}

\section{Methodology}

Adenosine 5'-monophosphate (AMP) sodium salt ( $\geq 99 \%$ purity) and uridine 5'monophosphate (UMP) disodium salt (>98\% purity) were purchase from Sigma-Aldrich and used as received. A $50 \mathrm{mM}$ phosphate buffered saline (PBS) solution was prepared using monosodium phosphate and disodium phosphate salts. The $\mathrm{pH}$ was adjusted using phosphoric acid (Fisher Chemical, certified ACS grade $\geq 85 \% \mathrm{w} / \mathrm{w}$ ) and $0.1 \mathrm{M}$ sodium hydroxide solutions to the desired $\mathrm{pH}$ value of $7.3 \pm 0.1$. The AMP and UMP solutions were made to have the same absorbance (2.20) at the excitation wavelength of $267 \mathrm{~nm}$, in order to perform successive experiments and to subtract any contributions from two-photon ionization of water to the transient spectra and kinetic traces. ${ }^{1}$ The correction for the two-photon ionization of water follows the procedure developed by Crespo-Hernández and Kohler. ${ }^{1}$

The experimental setup and data analysis have been discussed in detail elsewhere. ${ }^{2-4}$ Briefly, a Libra-HE (Coherent, Inc.) was used to generate the $800 \mathrm{~nm}$ laser beam at a $1 \mathrm{kHz}$ repetition rate with a pulse width of $100 \mathrm{fs}$. The fundamental beam was split in two beams using a 98/2 beam splitter. The low-power beam was used to generate white light in a translating calcium fluoride crystal with a spectral probe window from 320 to $700 \mathrm{~nm}$. The high-power fundamental beam was used to seed two $0.5 \mathrm{~mm}$ Barium borate crystals to generate a $400 \mathrm{~nm}$ light and $267 \mathrm{~nm}$ light. The generated $267 \mathrm{~nm}$ light was separated from the excess generated $400 \mathrm{~nm}$ and fundamental $800 \mathrm{~nm}$ light through the use of dichroic mirrors. Time zero was set to the maximum intensity of the transient absorption signal in the visible region of the nucleotide, which matched the maximum intensity of a negative amplitude band observed in the UMP transient data. The instrument response was estimated from the two photon absorption of pure methanol to be $300 \pm$ $50 \mathrm{fs} .{ }^{5}$ Homogeneity of the solutions in a $2 \mathrm{~mm}$ fused silica cuvette were maintained using a magnetic stir bar throughout the experiments. Freshly prepared solutions were used if the absorbance of the nucleotide at its lowest-energy absorption maximum decreases by more than 5 $\%$, as judged by steady-state absorption spectroscopy (Cary 100 Bio Instrument, Agilent Technologies). This procedure ensures that the transient absorption signals were not contaminated by signals from any putative formation of photoproducts.

Data analysis was performed using Igor Pro 6.37A software (Wavemetrics, Inc.). From a multidimensional data set, 60 decay traces were selected equidistant between probe wavelengths, and analyzed using a global fitting subroutine set up in the Igor Pro software. A global fit analysis 
method based on a sequential kinetic model and convoluted with an instrument response of $300 \mathrm{fs}$ was used to determine the global lifetimes determined from successive experiments for the nucleotide after solvent correction was performed, as mentioned above. The evolution associated difference spectra (EADS) were obtained from the target and global analyses. ${ }^{6}$

\section{Supporting Results}

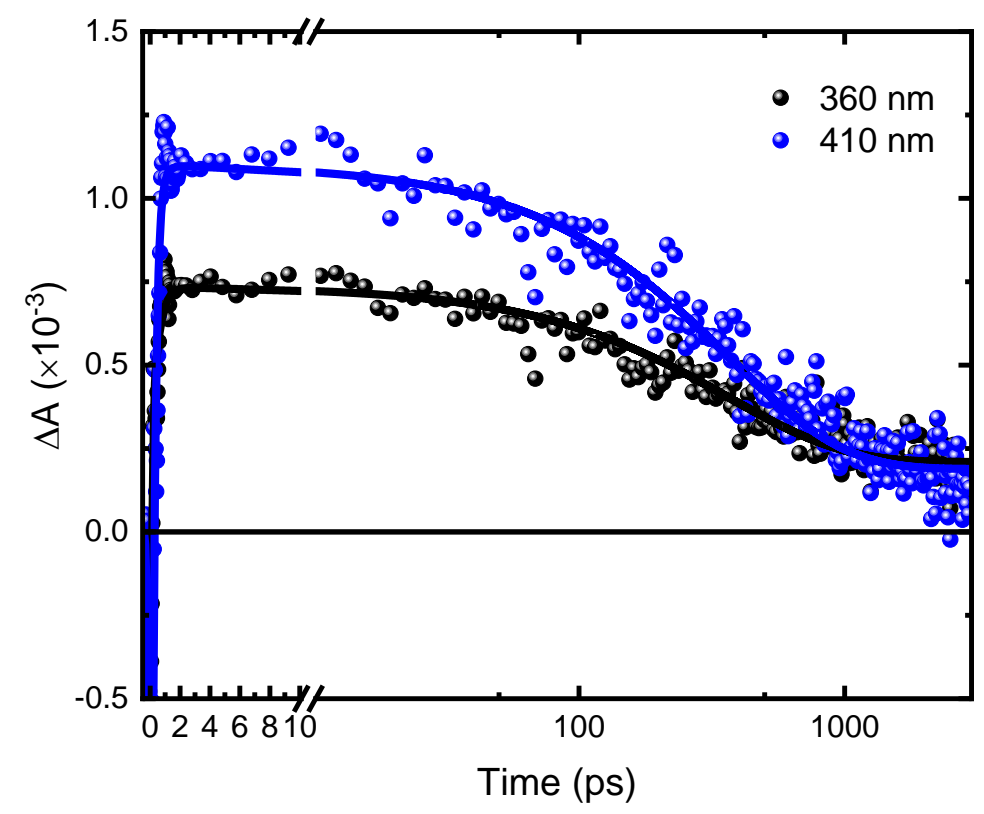

Figure S1. Kinetic traces at $360 \mathrm{~nm}$ and $410 \mathrm{~nm}$.

\section{References}

1. Crespo-Hernández, C. E.; Kohler, B. Influence of Secondary Structure on Electronic Energy Relaxation in Adenine Homopolymers. J. Phys. Chem. B 2004, 108, 11182-11188.

2. Reichardt, C.; Vogt, R. A.; Crespo-Hernández, C. E. On the Origin of Ultrafast Nonradiative Transitions in Nitro-Polycyclic Aromatic Hydrocarbons: Excited-State Dynamics in 1-Nitronaphthalene. J. Chem. Phys. 2009, 131, 224518.

3. Pollum, M.; Jockusch, S.; Crespo-Hernández, C. E. 2,4-Dithiothymine as a Potent UVA Chemotherapeutic Agent. J. Am. Chem. Soc. 2014, 136, 17930-17933.

4. Brister, M. M.; Crespo-Hernández, C. E. Direct Observation of Triplet-State Population Dynamics in the RNA Uracil Derivative 1-Cyclohexyluracil. J. Phys. Chem. Lett. 2015, 6, 44044409.

5. Rasmusson, M.; Tarnovsky, A. N.; Åkesson, E.; Sundström, V. On the Use of TwoPhoton Absorption for Determination of Femtosecond Pump-Probe Cross-Correlation Functions. Chem. Phys. Lett. 2001, 335, 201-208.

6. van Stokkum, I. H. M.; Larsen, D. S.; van Grondelle, R. Global and Target Analysis of Time-Resolved Spectra. Biochim. Biophys. Acta 2004, 1657, 82-104. 